\title{
Phase II, Open-Label, Randomized, Multicenter Trial (HERBY) of Bevacizumab in Pediatric Patients With Newly Diagnosed High-Grade Glioma
}

Jacques Grill, Maura Massimino, Eric Bouffet, Amedeo A. Azizi, Geoffrey McCowage, Adela Cañete, Frank Saran, Marie-Cécile Le Deley, Pascale Varlet, Paul S. Morgan, Tim Jaspan, Chris Jones, Felice Giangaspero, Helen Smith, Josep Garcia, Markus C. Elze, Raphaël F. Rousseau, Lauren Abrey, Darren Hargrave, and Gilles Vassal

Author affiliations and support information (if applicable) appear at the end of this article.

Published at jco.org on February 7, 2018 D.H. and G.V. contributed equally to this work.

This study was a partnership between Australasian Children's Cancer Trials, Innovative Therapies for Children with Cancer, the European Society for Paediatric Oncology, and Roche, and was part of a pediatric investigation plan requested by the European Medicines Agency

Clinical trial information: NCT01390948.

Corresponding author: Jacques Grill, MD, $\mathrm{PhD}$, Pediatric and Adolescent Oncology, Institut Gustave-Roussy, 114 Rue Edouard-Vaillant, 94805 Villejuif, Cedex France; e-mail: jacques.grill@ gustaveroussy.fr.

() 2018 by American Society of Clinical Oncology

0732-183X/18/3699-1/\$20.00

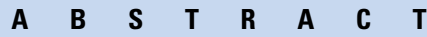

\section{Purpose}

Bevacizumab (BEV) is approved in more than 60 countries for use in adults with recurrent glioblastoma. We evaluated the addition of BEV to radiotherapy plus temozolomide (RT+TMZ) in pediatric patients with newly diagnosed high-grade glioma (HGG).

\section{Methods}

The randomized, parallel group, multicenter, open-label HERBY trial (ClinicalTrials.gov identifier: NCT01390948) enrolled patients age $\geq 3$ years to $\leq 18$ years with localized, centrally neuropathology-confirmed, nonbrainstem HGG. Eligible patients were randomly assigned to receive RT + TMZ (RT: $1.8 \mathrm{~Gy}, 5$ days per week, and TMZ: $75 \mathrm{mg} / \mathrm{m}^{2}$ per day for 6 weeks; 4-week treatment break; then up to $12 \times 28$-day cycles of TMZ [cycle $1: 150 \mathrm{mg} / \mathrm{m}^{2}$ per day, days 1 to 5 ; cycles 2 to 12 : $200 \mathrm{mg} / \mathrm{m}^{2}$ per day, days 1 to 5]) with or without BEV (10 mg/kg every 2 weeks). The primary end point was event-free survival (EFS) as assessed by a central radiology review committee that was blinded to treatment. We report findings of EFS at 12 months after the enrollment of the last patient.

\section{Results}

One hundred twenty-one patients were enrolled (RT+TMZ [n = 59]; BEV plus RT+TMZ [n = 62]). Central radiology review committee-assessed median EFS did not differ significantly between treatment groups (RT+TMZ, 11.8 months; $95 \% \mathrm{Cl}, 7.9$ to 16.4 months; $\mathrm{BEV}$ plus $\mathrm{RT}+\mathrm{TMZ}$, 8.2 months; $95 \% \mathrm{Cl}, 7.8$ to 12.7 months; hazard ratio, $1.44 ; P=.13$ [stratified log-rank test]). In the overall survival analysis, the addition of BEV did not reduce the risk of death (hazard ratio, $1.23 ; 95 \%$ $\mathrm{Cl}, 0.72$ to 2.09). More patients in the BEV plus RT+TMZ group versus the RT+TMZ group experienced one or more serious adverse events ( $n=35[58 \%] v n=27$ [48\%]), and more patients who received $B E V$ discontinued study treatment as a result of adverse events ( $n=13[22 \%] v n=3[5 \%]$ ).

\section{Conclusion}

Adding BEV to RT+TMZ did not improve EFS in pediatric patients with newly diagnosed HGG. Our findings were not comparable to those of previous adult trials, which highlights the importance of performing pediatric-specific studies.

\section{J Clin Oncol 36. (C) 2018 by American Society of Clinical Oncology}

\section{INTRODUCTION}

High-grade gliomas (HGGs) are the most common group of pediatric malignant CNS neoplasms, with an annual incidence of 0.87 per 100,000 children in the United States. ${ }^{1}$ Despite surgical resection followed by radiotherapy (RT) and concomitant adjuvant chemotherapy, the prognosis for children with HGG remains poor. Unlike most other cancer types, 5-year survival is lower in pediatric patients versus adult patients with $\mathrm{HGG}^{1,2}$
There are substantial differences between pediatric and adult $\mathrm{HGG}^{3-5}$ Midline tumor location is more frequent in children than in adults. ${ }^{6,7}$ Whereas contrast enhancement is the hallmark of malignant gliomas in adults, not all pediatric HGGs exhibit contrast uptake. ${ }^{8,9}$ Platelet-derived growth factor receptor- $\alpha$ amplification is the most common DNA copy number change in pediatric $\mathrm{HGG}$, whereas epidermal growth factor receptor amplification is more commonly detected in adults. ${ }^{5} \mathrm{O}^{6}$ methylguanine DNA methyltransferase (MGMT) 
promoter expression is also less frequent in pediatric patients compared with adult patients with HGG. ${ }^{10}$ A key difference in pediatric HGGs compared with adult HGGs is the presence of unique somatic $\mathrm{H} 3 \mathrm{~F} 3 \mathrm{~A}$ (histone H3.3) driver mutations at position $\mathrm{K} 27 \mathrm{M}$ and G34R/V of the regulatory tail, ${ }^{11}$ which suggests that results from adult trials may not be directly transferable to pediatric patients with the same histologically defined disease.

Bevacizumab (BEV) is approved in more than 60 countries worldwide for use in adults with recurrent glioblastoma. ${ }^{12,13}$ Clinical experience with BEV in pediatric patients with $\mathrm{HGG}$ is limited, although the addition of BEV to irinotecan in 31 children with recurrent malignant glioma or intrinsic brainstem glioma demonstrated some efficacy and was well tolerated. ${ }^{14}$ The HERBY trial aimed to evaluate the efficacy and safety of adding BEV to postoperative radiotherapy plus temozolomide (RT+TMZ) in pediatric patients with newly diagnosed, localized HGG.

\section{METHODS}

\section{Study Design}

A Study of Bevacizumab (Avastin) in Combination with Temozolomide (TMZ) and Radiotherapy in Paediatric and Adolescent Participants With High-Grade Giloma (HERBY) was a phase II, open-label, randomized, international, comparator study of the addition of BEV to RT + TMZ in pediatric patients (age $\geq 3$ to $\leq 18$ years) with newly diagnosed HGG. Here, we present event-free survival (EFS) data at 12 months after enrollment of the last patient.

HERBY was conducted as part of a pediatric investigation plan and in accordance with applicable country regulations, International Council for Harmonisation of Technical Requirements for Pharmaceuticals for Human Use Good Clinical Practice Guidelines, and the Declaration of Helsinki. Written informed consent was obtained from the patient, parents, or legally acceptable representatives before enrollment and the collection of tissue for exploratory biomarker analyses.

Eligible patients were centrally randomly assigned 1:1 on the basis of a minimization algorithm via an interactive voice response system to receive $\mathrm{BEV}$ plus $\mathrm{RT}+\mathrm{TMZ}$ or $\mathrm{RT}+\mathrm{TMZ}$ with the following stratification factors: age ( $\geq 3$ to $\leq 5$ years, $\geq 6$ to $\leq 12$ years, and $\geq 13$ to $\leq 18$ years), WHO grade (III $v$ IV), and type of surgery (total/near total resection $v$ others; Fig 1). Random assignment was performed via minimization with biased coin assignment. ${ }^{15}$ Patients and investigators were not masked to treatment assignment; the central radiology review committee (CRRC) was masked to group allocation.

Study treatment started at least 28 days after cranial surgery and no later than 6 weeks after the last major surgery. Patients received RT (1.8 Gy per session for 30 sessions, 5 days per week, for a total of 54 Gy) and TMZ $75 \mathrm{mg} / \mathrm{m}^{2}$ per day for 6 weeks, followed by a minimum 4-week TMZ treatment break, then up to $12 \times 28$-day cycles of TMZ (cycle $1: 150 \mathrm{mg} / \mathrm{m}^{2}$ per day, days 1 to 5; cycles 2 to $12: 200 \mathrm{mg} / \mathrm{m}^{2}$ per day, days 1 to 5 ). Patients who were assigned to BEV treatment additionally received BEV $10 \mathrm{mg} / \mathrm{kg}$ every 2 weeks, which was delivered concomitantly with RT+TMZ (concurrent phase), alone during the TMZ treatment break, and subsequently with up to $12 \times 28$-day cycles of TMZ (adjuvant phase). Concomitant corticosteroid use and stable doses of anticoagulants were permitted.

\section{Patients}

Patients aged $\geq 3$ to $\leq 18$ years with newly diagnosed, localized, supratentorial or infratentorial cerebellar or peduncular, grade III and IV gliomas (according to WHO 2007 guidelines) were enrolled. Local histologic diagnosis was confirmed by a central reference neuropathologist before enrollment. The availability of a baseline magnetic resonance imaging scan and the ability to commence trial treatment 4 to 6 weeks after surgery were also required. Key exclusion criteria included metastatic HGG defined as evidence of neuro-axis dissemination by magnetic resonance imaging or positive CSF cytology; gliomatosis cerebri (extensive glioma, ie, involving at least three cerebral lobes according to WHO 2007 guidelines), multifocal glioma, diffuse intrinsic pontine glioma, or intramedullary HGG; pleomorphic xanthoastrocytoma or anaplastic ganglioglioma; prior diagnosis of a malignancy, including low-grade glioma, and not disease free for 5 years; prior systemic anticancer therapy; previous cranial irradiation; any significant cardiovascular disease or unresolved infection; or chronic daily treatment with aspirin ( $>325 \mathrm{mg}$ per day) or clopidogrel ( $>75 \mathrm{mg}$ per day).

\section{Study Assessments}

Patients were observed for a minimum of 1 year after random assignment. Tumor progression and/or recurrence and BEV response were determined by using Response Assessment in Neuro-Oncology criteria in HGG. ${ }^{16}$ Tumor evaluations were performed at baseline, at the end of the TMZ break, every 3 months during the adjuvant phase and the first 3 years post-random assignment, and every 6 months thereafter until progression and/or recurrence. ${ }^{17}$

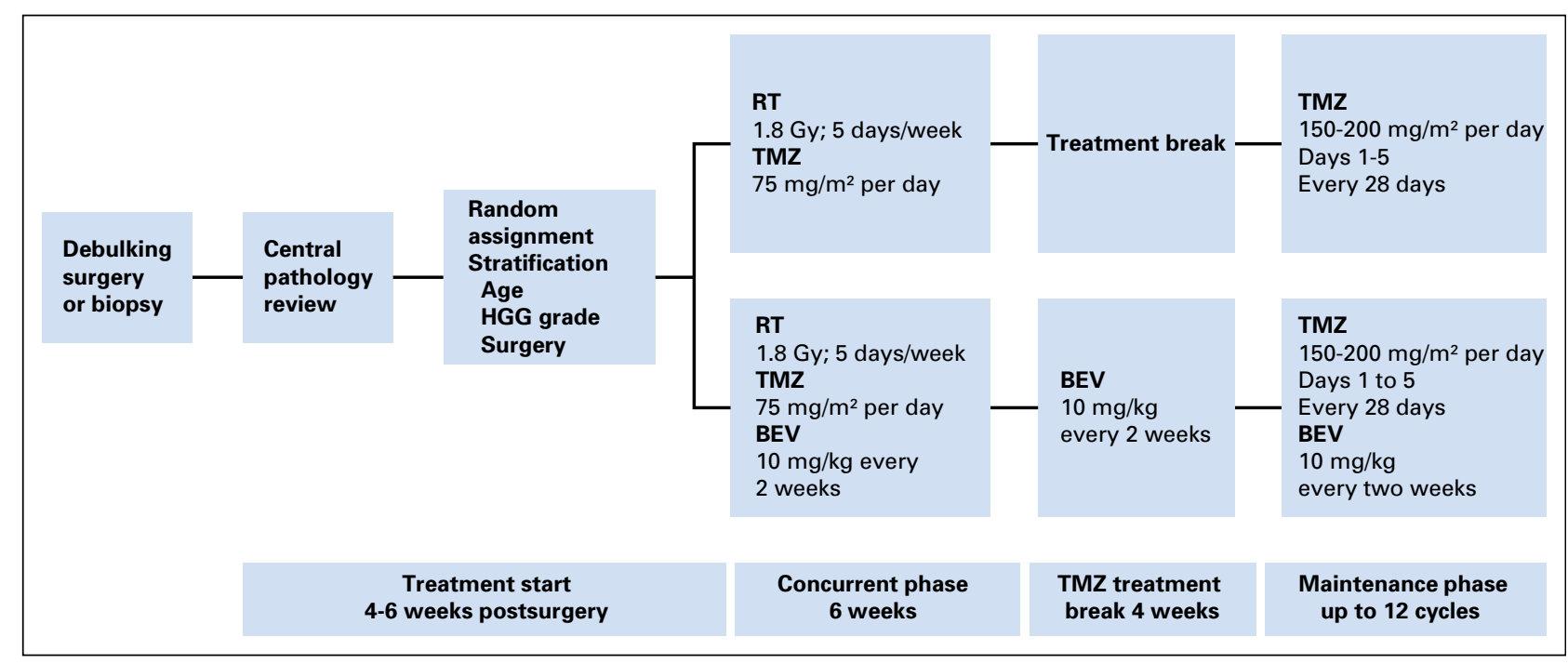

Fig 1. Study design. BEV, bevacizumab; HGG, high-grade glioma; RT, radiotherapy; TMZ, temozolomide. 
Health-related quality of life was assessed in patients $\geq 5$ years of age by using the Health Utility Index (HUI) questionnaire, ${ }^{18}$ which was completed at screening, at cycle 6 of the adjuvant phase, at the end of treatment, yearly during the follow-up period, at the time of progression, and at the end-of-study visit. Neuropsychological assessment using the Wechsler scale adapted for age was measured at the end of treatment, every 2 years during the follow-up period, and at the end-of-study visit.

\section{Study End Points}

EFS was the prespecified primary end point, which was defined as the earliest occurrence of tumor progression and tumor recurrence (CRRC assessed), second primary non-HGG malignancy, or death attributable to any cause. Prespecified secondary end points included overall survival (OS) and 1-year OS rate, 6-month and 1-year EFS rates (CRRC assessed), objective response rate (ORR; CRRC assessed using Response Assessment in Neuro-Oncology criteria), investigator-assessed EFS, health status as measured by HUI (patients age $\geq 5$ years), neuropsychological function as measured by the Wechsler scale adapted for age, and safety. We performed post hoc exploratory analyses of EFS (CRRC assessed) and OS by histone mutation status and tumor location.

\section{Safety}

Adverse events (AEs) and serious AEs (SAEs) were reported from study treatment initiation to 28 days after the last dose of the study treatment. AEs of special interest (AESIs), regardless of the relationship to the study treatment, were reported up to 6 months after the last dose of the study treatment. After 6 months, only study treatment-related SAEs were reported.

\section{Statistical Analysis}

Analysis populations included the intent-to-treat population (all randomly assigned patients regardless of whether they received study treatment), the efficacy-evaluable population (all randomly assigned patients with at least one post-random assignment assessment from the local investigator), and the safety-evaluable population (all randomly assigned patients who received at least one dose of the study treatment). Baseline characteristics were compared between treatment groups by using $\chi^{2}$, Mann-Whitney-Wilcoxon, or Kruskal-Wallis tests as appropriate.

The primary end point of CRRC-assessed EFS was estimated by using Kaplan-Meier methodology and compared between treatment groups by using a stratified log-rank test (two-sided) at a 5\% level of significance. Stratification factors were age, HGG WHO grade, and the extent of surgery. Estimates of treatment effect (BEV plus RT+TMZ $v$ RT+TMZ) stratified for covariates were expressed as hazard ratios (HRs) with 95\% CIs estimated in a Cox proportional hazards regression model. Investigator-assessed EFS and OS were analyzed by using a two-sided log-rank test. Safety data are described according to the maximum grade of intensity reported per preferred term, per patient.

Additional details on the study assessments, AESI definitions, and statistical analyses performed, including sample size calculations, interim futility analysis, and prespecified sensitivity analyses are available in the Appendix (online only).

\section{RESULTS}

\section{Patients}

Between October 2011 and February 2015, 174 patients were screened (53 patients were excluded at screening), and 121 were randomly assigned to receive treatment $(\mathrm{RT}+\mathrm{TMZ}[\mathrm{n}=59]$; BEV plus RT+TMZ $[\mathrm{n}=62]$; Fig 2). Overall, 116 patients (RT+TMZ $[\mathrm{n}=56]$; BEV plus $\mathrm{RT}+\mathrm{TMZ}[\mathrm{n}=60]$ ) received the study treatment at 51 sites in 14 countries.

Baseline characteristics were balanced, with no significant differences between treatment groups for any of the variables listed in Table 1. MGMT promotor status was assessed in 42 patients, of whom 37 had an unmethylated tumor (RT+TMZ [n = 18]; BEV plus RT+TMZ [n = 19]; Table 1). H3F3A mutation status was assessed in 85 patients, of whom 31 showed evidence of a mutation (RT+TMZ [ $\mathrm{n}=15]$; BEV plus RT+TMZ [ $\mathrm{n}=16]$; Table 1). Mutations were observed at position $\mathrm{K} 27 \mathrm{M}$ in 24 patients (RT+TMZ $[\mathrm{n}=10]$; BEV plus RT+TMZ $[\mathrm{n}=14])$ and at position $\mathrm{G} 34 \mathrm{R} / \mathrm{V}$ in seven patients (RT+TMZ $[\mathrm{n}=5]$; BEV plus $\mathrm{RT}+\mathrm{TMZ}[\mathrm{n}=2]$; Table 1). Additional MGMT promotor and histone mutation status findings from subsequent analyses using non-prespecified tests are reported in a separate paper (Mackay A, et al. [In submission]).

The median duration of survival follow-up was similar between treatment groups (RT+TMZ: 15.2 months; range, 0.1 to 46.8 months; and BEV plus RT+TMZ: 16.2 months; range, 0 to 45.7 months).

\section{Interim Analysis}

The study was considered futile after the prespecified interim analysis, which was performed after the first 60 randomly assigned patients were observed for 1 year; however, as patient recruitment had been completed, and there were no safety concerns by the time the interim analysis was performed, the independent data monitoring committee recommended continuing the treatment of ongoing patients per the protocol.

\section{Primary Efficacy End Point}

Median CRRC-assessed EFS for RT+TMZ and BEV plus RT+TMZ was 11.8 months (95\% CI, 7.9 to 16.4 months) and 8.2 months ( $95 \%$ CI, 7.8 to 12.7 months), respectively (stratified HR, 1.44; $95 \%$ CI, 0.90 to 2.30; $P=.13$; Fig 3 ). The earliest contributing event was tumor progression ( $\mathrm{RT}+\mathrm{TMZ}[\mathrm{n}=35]$; BEV plus $\mathrm{RT}+\mathrm{TMZ}$ $[\mathrm{n}=38])$, death (each group $[\mathrm{n}=3])$, tumor recurrence (RT+TMZ $[\mathrm{n}=1]$; BEV plus RT+TMZ $[\mathrm{n}=4])$, and second primary non-HGG malignancy (osteosarcoma [RT+TMZ] and B-cell acute lymphocytic leukemia [BEV plus RT+TMZ]; each group $[n=1])$. Results were generally consistent across the different subgroups (Fig 4), although females who received RT+TMZ demonstrated a better outcome than did those who received BEV plus RT+TMZ (HR, 2.10; 95\% CI, 1.04 to 4.21 ).

\section{Secondary Efficacy End Points}

The 1-year CRRC-assessed EFS rates were 48\% (95\% CI, 35\% to $61 \%$ ) and $38 \%$ (95\% CI, $26 \%$ to $51 \%$ ) for $\mathrm{RT}+\mathrm{TMZ}$ and $\mathrm{BEV}$ plus RT+TMZ, respectively. The 1-year OS rates were $68 \%$ (95\% CI, $54 \%$ to $78 \%$ ) and $75 \%$ (95\% CI, $61 \%$ to $84 \%$ ), respectively. As a result of the absence of measurable lesions at baseline, only 27 patients were eligible for CRRC-assessed ORR analysis. Among these patients, ORR was 40\% (six of 15 patients) and 42\% (five of 12 patients) in the RT+TMZ and BEV plus RT+TMZ groups, respectively. Results for investigator-assessed EFS (HR, 1.49; 95\% CI, 0.92 to 2.40) were similar to those for CRRC-assessed EFS. The addition of BEV did not reduce the risk of death (HR, 1.23; 95\% CI, 0.72 to 2.09; Fig 5). OS data are immature; a final OS analysis will be performed once 5 years of follow-up are available. The most common pattern of progression in both groups was local recurrence (RT+TMZ $[\mathrm{n}=25]$; BEV plus RT+TMZ [n=23]). More patients in the BEV plus $\mathrm{RT}+\mathrm{TMZ}$ group $(\mathrm{n}=15)$ than in the $\mathrm{RT}+\mathrm{TMZ}$ group $(\mathrm{n}=8)$ demonstrated both local and distant recurrence. 


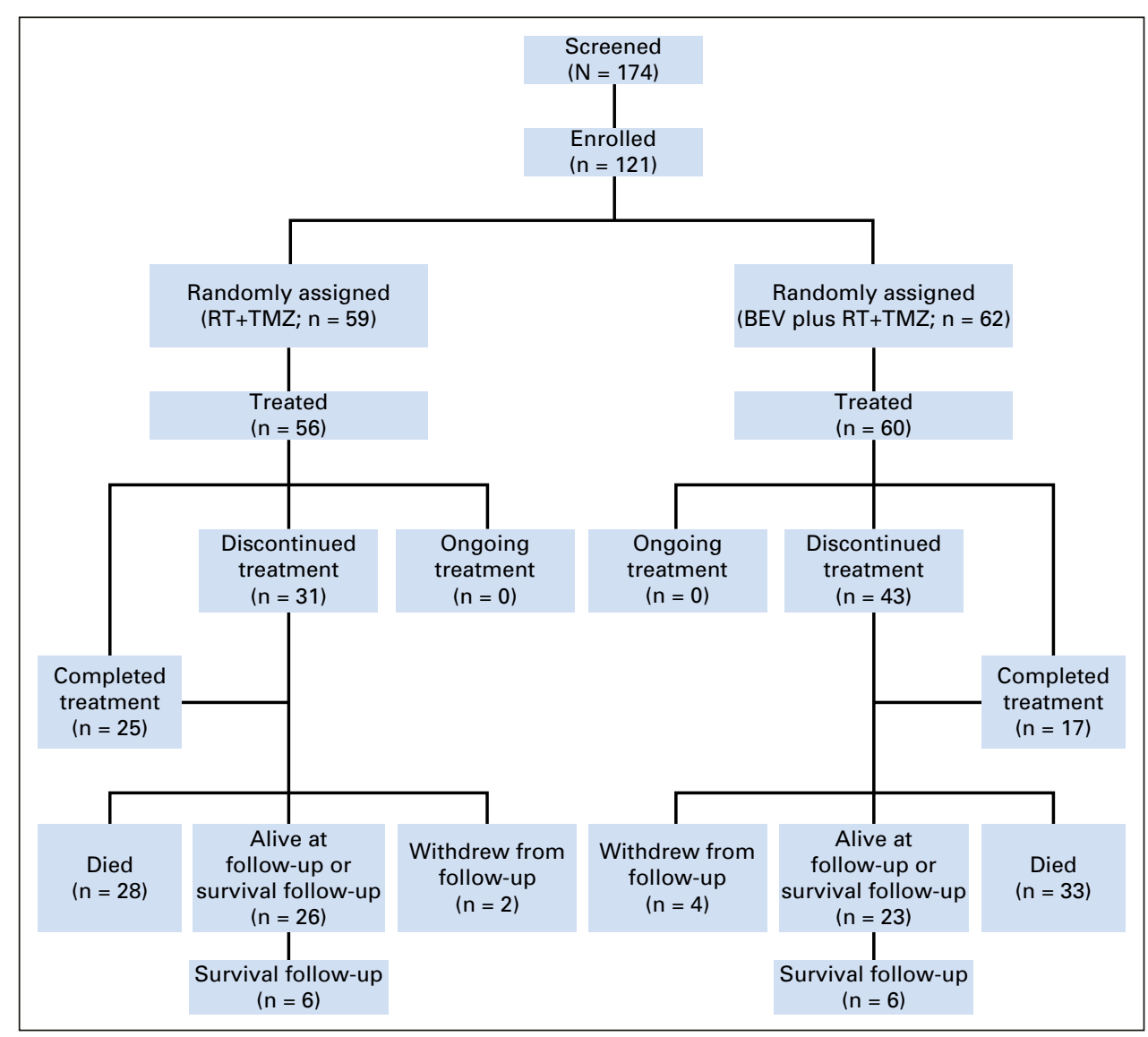

Fig 2. CONSORT flow diagram. Survival follow-up was an unlimited follow-up that continued to capture patient survival after regular follow-up had been completed. Five randomly assigned patients did not receive treatment (radiotherapy plus temozolomide [RT+TMZ]: withdrew consent [ $=3]$; bevacizumab [BEV] plus RT+TMZ: failed to meet eligibility criteria $[n=1]$; withdrew consent $[n=1])$.

\section{Treatment Received}

During the concurrent phase, $95 \%$ and $98 \%$ of patients treated with $\mathrm{RT}+\mathrm{TMZ}$ and $\mathrm{BEV}$ plus $\mathrm{RT}+\mathrm{TMZ}$, respectively, completed $\geq 90 \%$ of planned RT doses, and $86 \%$ and $88 \%$ of patients, respectively, completed $\geq 90 \%$ of planned TMZ doses. Correspondingly, during the adjuvant phase, $45 \%$ and $33 \%$ of patients, respectively, completed $12 \mathrm{TMZ}$ cycles. The total and percycle TMZ dose received was comparable between groups for the concurrent and adjuvant phases. Patients in the BEV plus RT +TMZ group received a median of 5.0 and $18.5 \mathrm{BEV}$ administrations during the concurrent and adjuvant phases, respectively.

\section{Safety}

The median duration of safety follow-up was 11.7 months and 11.6 months in the RT+TMZ and BEV plus RT+TMZ groups, respectively. No new safety signals were identified for BEV. All patients, with the exception of one in the BEV plus RT+TMZ group, experienced at least one AE. The incidence of grade 3 to 5 AEs was similar across the groups (RT+TMZ $[\mathrm{n}=38 ; 68 \%] v \mathrm{BEV}$ plus RT+TMZ [n $=42 ; 70 \%])$, but more patients experienced a grade 3 to 5 AESI in the BEV plus RT+TMZ group $(n=13 ; 22 \%)$ than in the RT+TMZ group $(\mathrm{n}=3 ; 5 \%)$. The most common AESIs were proteinuria $(\mathrm{RT}+\mathrm{TMZ}[\mathrm{n}=0 ; 0 \%] v \mathrm{BEV}$ plus $\mathrm{RT}+\mathrm{TMZ}$ $[\mathrm{n}=8 ; 13 \%])$ and arterial thromboembolic events (RT+TMZ $[\mathrm{n}=2$; $4 \%$ ] $v$ BEV plus RT+TMZ $[\mathrm{n}=5 ; 8 \%])$. More patients experienced at least one SAE in the BEV plus RT+TMZ group $(\mathrm{n}=35 ; 58 \%)$ than in the RT+TMZ group $(\mathrm{n}=27 ; 48 \%)$, and a higher proportion of patients discontinued any component of study treatment as a result of AEs in the BEV plus RT+TMZ group $(n=13$; $22 \%)$ than in the RT+TMZ group $(n=3 ; 5 \%)$. More patients in the BEV plus RT+TMZ group $(\mathrm{n}=43 ; 72 \%)$ experienced AEs that led to dose modifications of any component of study treatment than in the RT+TMZ group $(\mathrm{n}=34 ; 61 \%)$.

In the BEV plus RT+TMZ group, BEV and TMZ were discontinued as a result of AEs in $20 \%$ and $5 \%$ of patients, respectively. Among patients who discontinued BEV because of an $\mathrm{AE}$, the most common reason was proteinuria $(n=6 ; 10 \%)$. At the clinical cutoff date, four of the proteinuria events had resolved after BEV discontinuation, and two were ongoing.

Deaths occurred in 28 patients $(50 \%)$ in the RT+TMZ group and 33 patients $(55 \%)$ in the BEV plus RT+TMZ group. The cause of death in all but one patient was disease progression. One treatmentrelated grade $4 \mathrm{AE}$ of atypical teratoid/rhabdoid tumor of the CNS occurred in the BEV plus RT+TMZ group 2 years after the end of the study treatment and resulted in death. Additional details on patient disposition and protocol deviations in this study, and results of the interim futility analysis, sensitivity analyses, health-related quality of life and neuropsychological function assessments, and exploratory analyses of potential prognostic factors are available in the Appendix.

\section{DISCUSSION}

The HERBY study evaluated the efficacy and safety of BEV plus $\mathrm{RT}+\mathrm{TMZ}$ versus $\mathrm{RT}+\mathrm{TMZ}$ alone in pediatric patients with newly 


\begin{tabular}{|c|c|c|c|}
\hline Characteristic & $\begin{array}{l}\mathrm{RT}+\mathrm{TMZ} \\
(\mathrm{n}=59)\end{array}$ & $\begin{array}{l}\text { BEV Plus RT+TMZ } \\
\quad(n=62)\end{array}$ & $\begin{array}{c}\text { Total } \\
(\mathrm{n}=121)\end{array}$ \\
\hline Median age (range), years & $11.0(3-17)$ & $10.0(3-17)$ & $11.0(3-17)$ \\
\hline 3 to $\leq 5$ & $6(10)$ & $10(16)$ & $16(13)$ \\
\hline 6 to $\leq 12$ & $30(51)$ & $35(57)$ & $65(54)$ \\
\hline 13 to $\leq 18$ & $23(39)$ & $17(27)$ & $40(33)$ \\
\hline III & $17(29)$ & $20(32)$ & $37(31)$ \\
\hline IV & $42(71)$ & $42(68)$ & $84(69)$ \\
\hline \multicolumn{4}{|l|}{ Surgery, No. $(\%)^{*}$} \\
\hline Total/near total resection & $29(49)$ & $31(50)$ & $60(50)$ \\
\hline Other resection & $20(34)$ & $19(31)$ & $39(32)$ \\
\hline Biopsy & $10(17)$ & $12(19)$ & $22(18)$ \\
\hline \multicolumn{4}{|l|}{ MGMT gene promotor status, No. (\%) } \\
\hline Mutation at position G34 & $5(9)$ & $2(3)$ & 7 (6) \\
\hline Mutation at position K27 & $10(17)$ & $14(23)$ & $24(20)$ \\
\hline Missing & $15(25)$ & $21(34)$ & $36(30)$ \\
\hline \multicolumn{4}{|l|}{ Location of HGG, No. (\%) } \\
\hline Midline & $18(31)$ & $24(39)$ & $42(35)$ \\
\hline Other & $41(69)$ & $38(61)$ & $79(65)$ \\
\hline \multicolumn{4}{|l|}{ Residual tumor at baseline, No. (\%)† } \\
\hline Contrast-enhancing lesions & $15(25)$ & $12(19)$ & $27(22)$ \\
\hline Non-contrast-enhancing lesions & $47(80)$ & 49 (79) & 96 (79) \\
\hline
\end{tabular}

Abbreviations: BEV, bevacizumab; HGG, high-grade glioma; MGMT, O ${ }^{6}$-methyguanine-DNA methyltransferase; RT, radiotherapy; TMZ, temozolomide.

* Stratification factors for random assignment.

tPatients could have both enhancing and nonenhancing lesions.

diagnosed nonbrainstem HGG. On the basis of a prespecified interim analysis of the first 60 randomly assigned patients who were observed for 1 year, the study was considered futile; however, as patient recruitment had been completed and there were no safety concerns, the independent data monitoring committee recommended the continued treatment of patients per the protocol. This work presents the updated analysis of the enrolled 121 patients in the main protocol who were observed for at least 1 year after random assignment, unless patient withdrawal or death occurred.

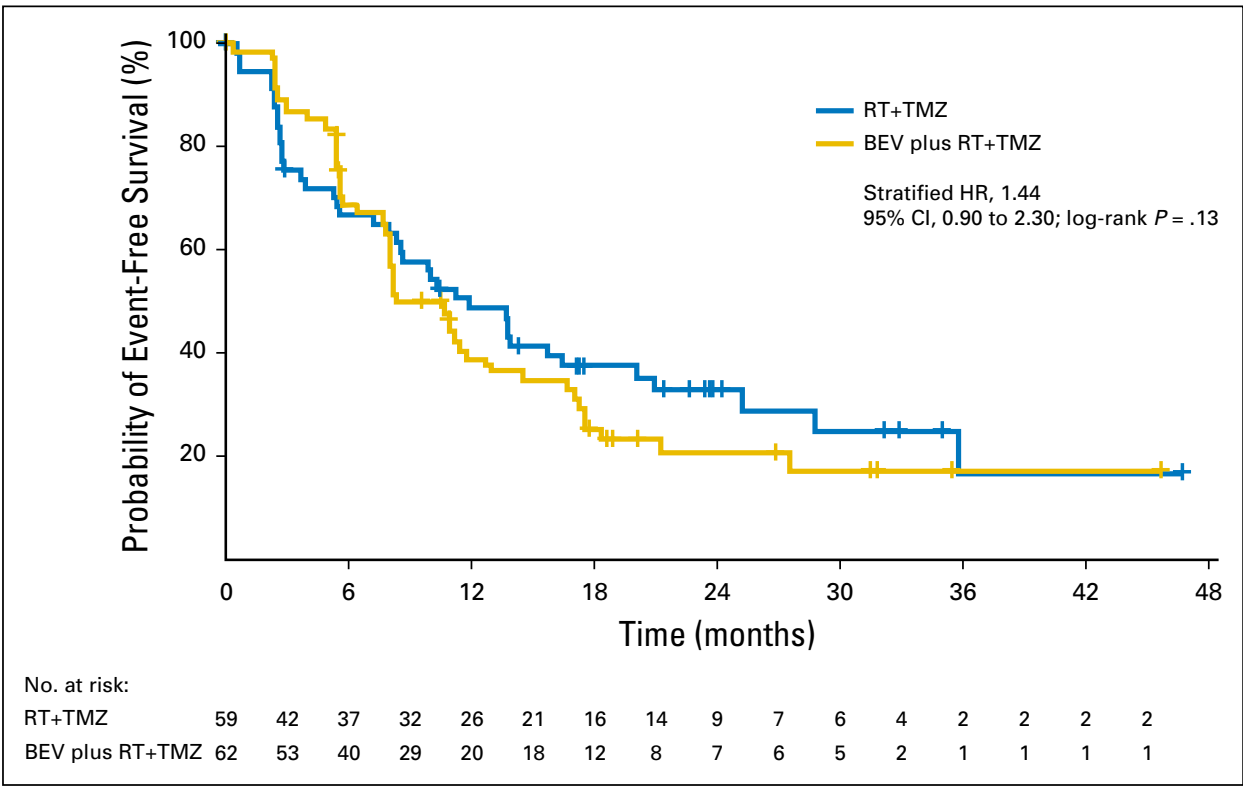

jco.org
Fig 3. Central radiology review committeeassessed event-free survival with radiotherapy plus temozolomide (RT+TMZ) and bevacizumab (BEV) plus RT+TMZ (primary efficacy end point). $H R$, hazard ratio. 


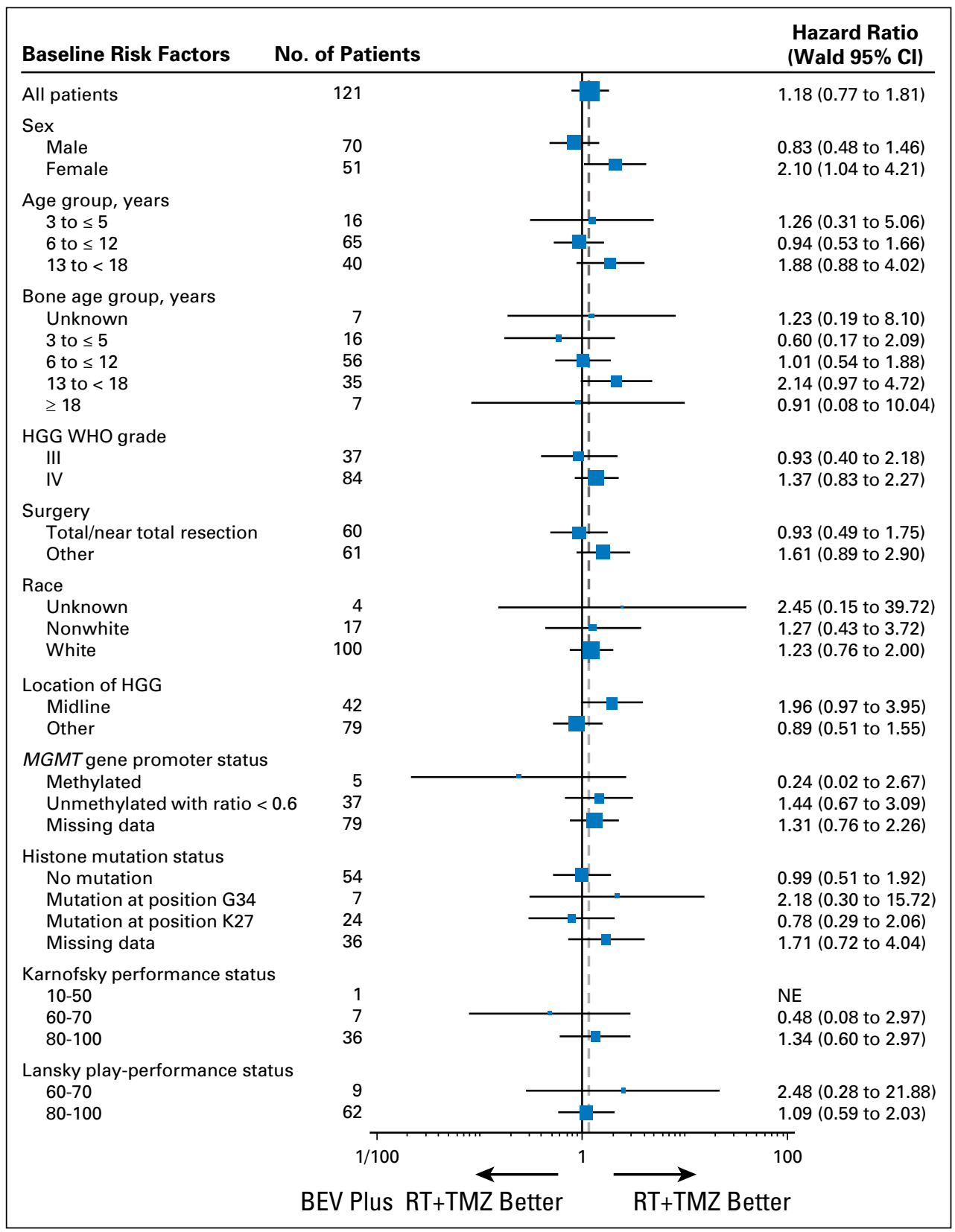

Fig 4. Forest plot of central radiology review committee-assessed event-free survival for the overall cohort and subgroups. BEV, bevacizumab; HGG, high-grade glioma; MGMT, $0^{6}$-methyguanine-DNA methyltransferase: $N E$, not evaluable; $R T$, radiotherapy; $T M Z$, temozolomide.
There was no significant difference in CRRC-assessed EFS - the primary end point-between treatment groups, and the results of the secondary end points, including investigator-assessed EFS, ORR, and OS, demonstrated no improvement with the addition of BEV. No new safety signals were identified for BEV; however, a higher proportion of patients in the BEV plus RT+TMZ group than in the RT+TMZ group discontinued study treatment as a result of toxicity. Patients in the RT+TMZ group had a higher-than-expected 1-year EFS rate of $48 \%$ (95\% CI, $35 \%$ to $61 \%$ ), which is comparable to a previously reported 1-year EFS rate of $38 \%$ with $\mathrm{RT}+\mathrm{TMZ}^{2}$ and $49 \%$ with TMZ plus lomustine. ${ }^{19}$

The absence of an EFS benefit with BEV in our study is not consistent with results from adult trials in which BEV has been shown to delay radiologic progression, although neither adult study demonstrated an OS benefit. ${ }^{20,21}$ Biologic differences between pediatric and adult HGGs may explain, in part, why children respond differently to treatments. ${ }^{3,4}$ Most patients in our study had non-contrast-enhancing lesions at baseline (79\%), whereas adult HGGs are typically contrast enhancing. ${ }^{8}$ In addition, the proportion of patients in our study with MGMT unmethylated tumors was lower than that reported in adult patients with $\mathrm{HGG},{ }^{10}$ which suggests a phenotypic difference. This highlights the importance of conducting pediatric-specific HGG trials to assess the benefit of potential treatments.

Between-group differences in tumor location may also have contributed to the lower-than-anticipated efficacy observed with $\mathrm{BEV}-39 \%$ of patients in the BEV plus RT $+\mathrm{TMZ}$ group had midline tumors versus $31 \%$ in the RT+TMZ group. Indeed, a previous study 


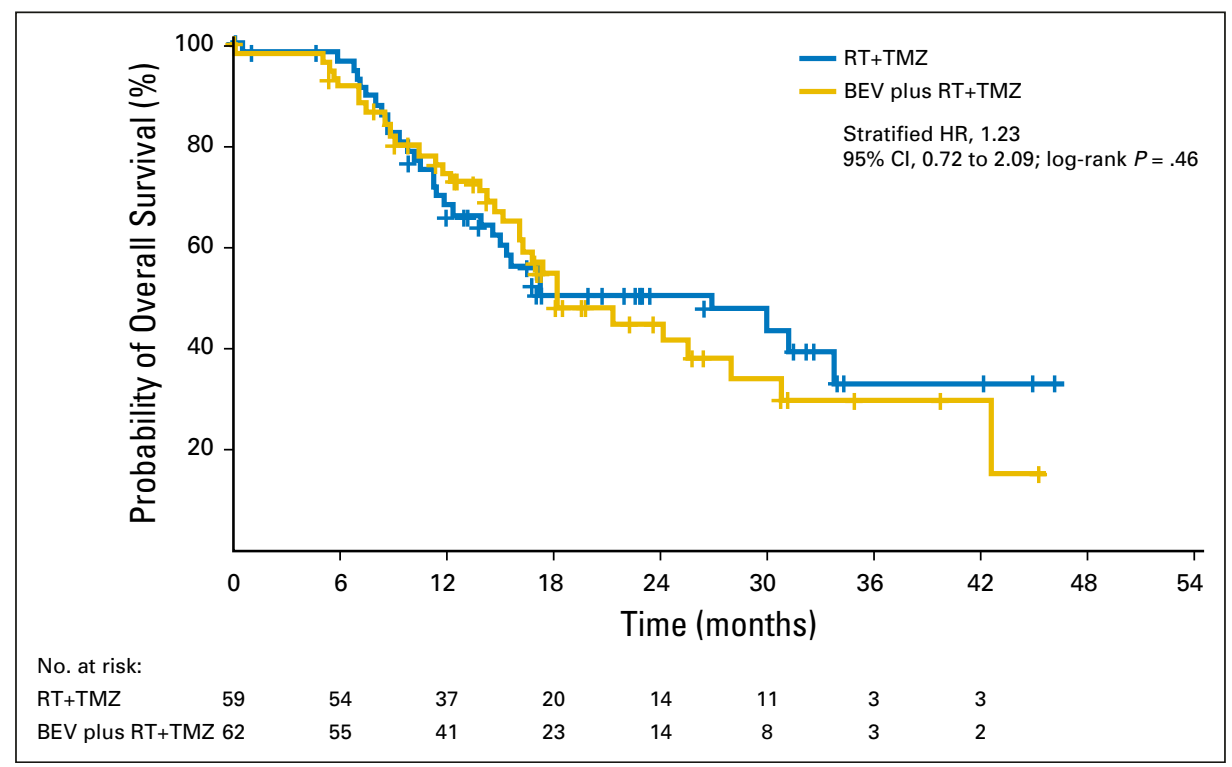

Fig 5. Probability of overall survival with radiotherapy plus temozolomide (RT+TMZ) and bevacizumab (BEV) plus RT+TMZ (interim assessment). HR, hazard ratio.

demonstrated poorer outcomes in patients with midline tumors versus other locations, although the study did not describe how midline was defined. ${ }^{22}$ In the current study, midline location was associated with poorer EFS than were tumors in other locations (Appendix). In addition, the effect of some biologic prognostic factors that were not anticipated at the time of the study design may have affected survival. Histone H3 K27M mutations, which are observed in midline tumors, have been associated with a poor prognosis, whereas mutations at G34R/V, observed in hemispheric tumors, may be associated with slightly longer OS. ${ }^{23}$ In our study, $\mathrm{H} 3 \mathrm{~F} 3 \mathrm{~A}$ driver mutations at position $\mathrm{K} 27 \mathrm{M}$ and G34M were associated with lower survival (Appendix). Some differences in the proportion of patients with these mutations were evident between groups; however, this finding should be interpreted with caution as multiple statistical tests were performed in a relatively small number of samples. MGMT methylation status has also been shown to influence response to $\mathrm{TMZ}^{24}$; however, MGMT methylation was relatively balanced between groups for the relatively small number of patients who were assessed for MGMT methylation status in this study.

The most common pattern of progressive disease in both groups was local recurrence, although a greater proportion of patients who received $\mathrm{BEV}$ demonstrated both local and distant progression. Previous research has suggested that BEV may lead to a higher incidence of distant and diffuse disease in pediatric patients with HGG or diffuse intrinsic pontine glioma, ${ }^{25}$ and adult studies have noted a greater proportion of distant lesions in patients who received $\mathrm{BEV}{ }^{26}$ although other studies reported no change in the radiographic pattern of patients' tumors between baseline and the time of disease progression in patients who received $\mathrm{BEV} .^{27}$

Limitations of this study include the heterogeneity of enrolled patients, the relatively short follow-up duration, and the low completion rate of the HUI questionnaire at follow-up. The statistical power of the study was limited by the relatively small number of enrolled patients; however, increasing the study sample size would be unlikely to change the point estimate for survival, but may reduce the associated CI. Despite this, the HERBY trial is one of the largest prospective, randomized pediatric HGG trials to date, including a molecular evaluation of tumor characteristics and providing a global picture of treatment efficacy. We have demonstrated the feasibility of real-time, central histopathologic review before study entry, with no delay in treatment initiation. Only 5\% of patients were excluded for having noneligible low-grade glioma compared with up to $30 \%$ in a trial that used post hoc central review. ${ }^{27}$ The availability of results within 4 years of the adult trial demonstrates successful pharma-academic cooperation.

In conclusion, adding BEV to RT+TMZ did not improve EFS in pediatric patients with newly diagnosed HGG. These results are not fully consistent with adult studies and highlight the biologic differences between adult and childhood HGG, as well as the importance of performing pediatric-specific studies.

\section{AUTHORS' DISCLOSURES OF POTENTIAL CONFLICTS OF INTEREST}

Disclosures provided by the authors are available with this article at jco.org.

\section{AUTHOR CONTRIBUTIONS}

Conception and design: Jacques Grill, Maura Massimino, Eric Bouffet, Amedeo A. Azizi, Adela Cañete, Frank Saran, Marie-Cécile Le Deley, Pascale Varlet, Paul S. Morgan, Tim Jaspan, Chris Jones, Felice Giangaspero, Helen Smith, Josep Garcia, Markus C. Elze, Raphaël F. Rousseau, Lauren Abrey, Darren Hargrave, Gilles Vassal

Collection and data analysis: Paul S. Morgan, Helen Smith, Markus C. Elze

Data analysis and interpretation: All authors

Manuscript writing: All authors

Final approval of manuscript: All authors

Accountable for all aspects of the work: All authors 


\section{REFERENCES}

1. Ostrom $\mathrm{QT}$, Gittleman $\mathrm{H}$, Farah $\mathrm{P}$, et al: CBTRUS statistical report: Primary brain and central nervous system tumors diagnosed in the United States in 2006-2010. Neuro Oncol 15:ii1-ii56, 2013 (suppl 2)

2. Cohen KJ, Pollack IF, Zhou T, et al: Temozolomide in the treatment of high-grade gliomas in children: A report from the Children's Oncology Group. Neuro Oncol 13:317-323, 2011

3. Jones C, Karajannis MA, Jones DTW, et al: Pediatric high-grade glioma: Biologically and clinically in need of new thinking. Neuro Oncol 19:153-161, 2017

4. Diaz AK, Baker SJ: The genetic signatures of pediatric high-grade glioma: No longer a one-act play. Semin Radiat Oncol 24:240-247, 2014

5. MacDonald TJ, Aguilera D, Kramm CM: Treatment of high-grade glioma in children and adolescents. Neuro Oncol 13:1049-1058, 2011

6. Puget S, Philippe C, Bax DA, et al: Mesenchymal transition and PDGFRA amplification/mutation are key distinct oncogenic events in pediatric diffuse intrinsic pontine gliomas. PLoS One 7:e30313, 2012

7. Baker SJ, Ellison DW, Gutmann DH: Pediatric gliomas as neurodevelopmental disorders. Glia 64: 879-895, 2016

8. Rees JH, Smirniotopoulos JG, Jones RV, et al: Glioblastoma multiforme: Radiologic-pathologic correlation. Radiographics 16:1413-1438, quiz 14621463, 1996

9. Cage TA, Mueller S, Haas-Kogan D, et al: Highgrade gliomas in children. Neurosurg Clin N Am 23: 515-523, 2012

10. Buttarelli FR, Massimino $M$, Antonelli $M$, et al: Evaluation status and prognostic significance of $\mathrm{O}^{6}$-methylguanine-DNA methyltransferase (MGMT) promoter methylation in pediatric high grade gliomas. Childs Nerv Syst 26:1051-1056, 2010

11. Schwartzentruber J, Korshunov A, Liu $X Y$, et al: Driver mutations in histone H3.3 and chromatin remodelling genes in paediatric glioblastoma. Nature 482:226-231, 2012

12. Cohen $M H$, Shen $Y L$, Keegan $P$, et al: FDA drug approval summary: Bevacizumab (Avastin) as treatment of recurrent glioblastoma multiforme. Oncologist 14:1131-1138, 2009

13. Friedman HS, Prados MD, Wen PY, et al: Bevacizumab alone and in combination with irinotecan in recurrent glioblastoma. J Clin Oncol 27: 4733-4740, 2009

14. Gururangan $S$, Chi SN, Young Poussaint $T$, et al: Lack of efficacy of bevacizumab plus irinotecan in children with recurrent malignant glioma and diffuse brainstem glioma: A Pediatric Brain Tumor Consortium study. J Clin Oncol 28:3069-3075, 2010

15. Pocock SJ, Simon R: Sequential treatment assignment with balancing for prognostic factors in the controlled clinical trial. Biometrics 31:103-115, 1975

16. Wen PY, Macdonald DR, Reardon DA, et al: Updated response assessment criteria for high-grade gliomas: response assessment in neuro-oncology working group. J Clin Oncol 28:1963-1972, 2010

17. Jaspan T, Morgan PS, Warmuth-Metz M, et al: Response assessment in pediatric neuro-oncology: Implementation and expansion of the RANO criteria in a randomized phase II trial of pediatric patients with newly diagnosed high-grade gliomas. AJNR Am J Neuroradiol 37:1581-1587, 2016

18. Feeny $D$, Furlong $W$, Torrance GW, et al: Multiattribute and single-attribute utility functions for the health utilities index mark 3 system. Med Care 40:113-128, 2002

19. Jakacki RI, Cohen KJ, Buxton A, et al: Phase 2 study of concurrent radiotherapy and temozolomide followed by temozolomide and lomustine in the treatment of children with high-grade glioma: $\mathrm{A}$ report of the Children's Oncology Group ACNS0423 study. Neuro Oncol 18:1442-1450, 2016

20. Chinot OL, Wick W, Mason W, et al: Bevacizumab plus radiotherapy-temozolomide for newly diagnosed glioblastoma. N Engl J Med 370:709-722, 2014

21. Gilbert MR, Dignam JJ, Armstrong TS, et al: A randomized trial of bevacizumab for newly diagnosed glioblastoma. N Engl J Med 370:699-708, 2014

22. Eisenstat DD, Pollack IF, Demers $A$, et al: Impact of tumor location and pathological discordance on survival of children with midline high-grade gliomas treated on Children's Cancer Group highgrade glioma study CCG-945. J Neurooncol 121: 573-581, 2015

23. Sturm D, Witt $H$, Hovestadt $V$, et al: Hotspot mutations in H3F3A and IDH1 define distinct epigenetic and biological subgroups of glioblastoma. Cancer Cell 22:425-437, 2012

24. Hegi ME, Diserens AC, Gorlia T, et al: MGMT gene silencing and benefit from temozolomide in glioblastoma. N Engl J Med 352: 997-1003, 2005

25. Salloum R, DeWire M, Lane A, et al: Patterns of progression in pediatric patients with high-grade glioma or diffuse intrinsic pontine glioma treated with bevacizumab-based therapy at diagnosis. J Neurooncol 121:591-598, 2015

26. Norden AD, Young GS, Setayesh $K$, et al: Bevacizumab for recurrent malignant gliomas: Efficacy, toxicity, and patterns of recurrence. Neurology 70:779-787, 2008

27. Pope WB, Xia Q, Paton VE, et al: Patterns of progression in patients with recurrent glioblastoma treated with bevacizumab. Neurology 76:432-437, 2011

\section{Affiliations}

Jacques Grill, Marie-Cécile Le Deley, and Gilles Vassal, Institut Gustave-Roussy, Villejuif; Marie-Cécile Le Deley, Paris-Saclay and Paris-Sud Universities, CESP, Institut National de la Santé et de la Recherche Médicale, Orsay; Pascale Varlet, Sainte-Anne Hospital, Paris, France; Maura Massimino, Fondazione Istituto di Ricovero e Cura a Carattere Scientifico (IRCCS) Istituto Nazionale dei Tumori, Milan; Felice Giangaspero, Sapienza University, Rome; Felice Giangaspero, IRCCS Neuromed, Pozzilli, Italy; Eric Bouffet, Hospital for Sick Children, Toronto, Ontario, Canada; Amedeo A. Azizi, Medical University of Vienna, Vienna, Austria; Geoffrey McCowage, Australasian Children's Cancer Trials, Clayton, Victoria, Australia; Adela Cañete, Hospital La Fe, Valencia, Spain; Frank Saran, The Royal Marsden Hospital; Chris Jones, The Institute of Cancer Research; Darren Hargrave, Great Ormond Street Hospital, London; Paul S. Morgan and Tim Jaspan, Nottingham University Hospitals, Queen's Medical Centre, Nottingham, United Kingdom; Helen Smith, Josep Garcia, Markus C. Elze, and Lauren Abrey, F. Hoffmann-La Roche Ltd, Basel, Switzerland; and Raphaël F. Rousseau, Gritstone Oncology, Emeryville, CA.

\section{Support}

Funded by F. Hoffmann-La Roche Ltd (Study No. BO25041). D.H. is supported by the National Institute for Health Research (NIHR) Biomedical Research Centre at Great Ormond Street Hospital for Children National Health Service (NHS) Foundation Trust and University College London. C.J. acknowledges NHS funding to the NIHR Biomedical Research Centre at The Royal Marsden Hospital and The Institute of Cancer Research.

\section{Prior Presentation}

Presented at the 17th International Symposium on Pediatric Neuro-Oncology, Liverpool, United Kingdom, June 12, 2016, the 48th Congress of the International Society of Paediatric Oncology, Dublin, Ireland, October 19-22, 2016, and the 21st Society for NeuroOncology Annual Scientific Meeting, Scottsdale, AZ, November 17-20, 2016. 


\section{AUTHORS' DISCLOSURES OF POTENTIAL CONFLICTS OF INTEREST}

Phase II, Open-Label, Randomized, Multicenter Trial (HERBY) of Bevacizumab in Pediatric Patients With Newly Diagnosed High-Grade Glioma

The following represents disclosure information provided by authors of this manuscript. All relationships are considered compensated. Relationships are self-held unless noted. I = Immediate Family Member, Inst = My Institution. Relationships may not relate to the subject matter of this manuscript. For more information about ASCO's conflict of interest policy, please refer to www.asco.org/rwc or ascopubs.org/jco/site/ifc.

Jacques Grill

Consulting or Advisory Role: F. Hoffmann-La Roche Ltd

Research Funding: Novartis, F. Hoffmann-La Roche Ltd, Bristol-Myers Squibb

\section{Maura Massimino}

Consulting or Advisory Role: Genentech, Roche

Eric Bouffet

Research Funding: F. Hoffmann-La Roche Ltd (Inst), Bristol-Myers Squibb (Inst)

\section{Amedeo A. Azizi}

Consulting or Advisory Role: F. Hoffmann-La Roche Ltd, AstraZeneca Travel, Accommodations, Expenses: F. Hoffmann-La Roche Ltd

\section{Geoffrey McCowage}

Consulting or Advisory Role: F. Hoffmann-La Roche Ltd Speakers' Bureau: F. Hoffmann-La Roche Ltd

Research Funding: Merck (Inst), Novartis (Inst), Epizyme (Inst)

Travel, Accommodations, Expenses: F. Hoffmann-La Roche Ltd

\section{Adela Cañete}

Consulting or Advisory Role: F. Hoffmann-La Roche Ltd

\section{Frank Saran}

Honoraria: Roche (I)

Consulting or Advisory Role: F. Hoffmann-La Roche Ltd, Bristol-Myers Squibb, Abbott, AbbVie

\section{Marie-Cécile Le Deley}

Consulting or Advisory Role: F. Hoffmann-La Roche Ltd

Pascale Varlet

Consulting or Advisory Role: F. Hoffmann-La Roche Ltd, Boehringer Ingelheim, Novartis, Pfizer

Research Funding: Novartis, F. Hoffmann-La Roche Ltd, Boehringer Ingelheim

Paul S. Morgan

Research Funding: F. Hoffmann-La Roche Ltd

Tim Jaspan

Consulting or Advisory Role: F. Hoffmann-La Roche Ltd

Research Funding: F. Hoffmann-La Roche Ltd
Chris Jones

Consulting or Advisory Role: F. Hoffmann-La Roche Ltd

Research Funding: F. Hoffmann-La Roche Ltd

Travel, Accommodations, Expenses: F. Hoffmann-La Roche Ltd

Felice Giangaspero

No relationship to disclose

Helen Smith

Employment: F. Hoffmann-La Roche Ltd

Stock or Other Ownership: F. Hoffmann-La Roche Ltd

Josep Garcia

Employment: F. Hoffmann-La Roche Ltd

Stock or Other Ownership: F. Hoffmann-La Roche Ltd

Markus C. Elze

Employment: F. Hoffmann-La Roche Ltd

Stock or Other Ownership: F. Hoffmann-La Roche Ltd

Travel, Accommodations, Expenses: F. Hoffmann-La Roche Ltd

Raphaël F. Rousseau

Employment: Genentech

Leadership: Gritstone Oncology

Stock or Other Ownership: Genentech, Gritstone Oncology

Lauren Abrey

Employment: F. Hoffmann-La Roche Ltd

Stock or Other Ownership: F. Hoffmann-La Roche Ltd

Darren Hargrave

Consulting or Advisory Role: AstraZeneca, Genentech, Novartis, Bayer, Boehringer Ingelheim

Research Funding: AstraZeneca

Expert Testimony: AstraZeneca

Travel, Accommodations, Expenses: Boehringer Ingelheim, Novartis, Genentech

Other Relationship: Celgene, Novartis, Bristol-Myers Squibb, Epizyme, AbbVie

\section{Gilles Vassal}

Consulting or Advisory Role: F. Hoffmann-La Roche Ltd, Bayer, Boehringer Ingelheim, Bristol-Myers Squibb, Celgene, Eli Lilly, Novartis, Pfizer 


\section{Acknowledgment}

We thank the participating investigators, their study staff, and the patients and their families who participated in the study. We also thank Magalie Hilton for contributing to the statistical analyses. Medical writing support in the form of the development of a draft manuscript was provided by Thomas Burton, BMBS, and David Evans, PhD, of Gardiner-Caldwell Communications (Macclesfield, United Kingdom) and was funded by F. Hoffmann-La Roche Ltd.

\section{Appendix}

\section{METHODS}

\section{Study Assessments}

The central radiology review committee (CRRC) performed a prospective review in patients with investigator-determined radiologic progression at first or second magnetic resonance imaging scan to ensure that discontinuation was not prematurely decided as a result of pseudoprogression, defined as a non-tumor-related increase in contrast enhancement observed on magnetic resonance imaging scan, typically occurring within the first 12 weeks after concomitant radiotherapy plus temozolomide (RT+TMZ), that then stabilizes or decreases over time in the absence of treatment modification. If subsequent imaging showed progression, the time of progression was backdated. A central efficacy read was performed by two independent radiologists in parallel and adjudicated when needed. In addition, the adjudicated radiology data were reviewed alongside clinical data by an independent oncologist. Objective response rate-a complete or partial response ( $>50 \%)$ on two consecutive occasions $\geq 4$ weeks apart—was evaluated in patients with measurable disease at baseline and based on the blinded evaluation by the CRRC using Response Assessment in Neuro-Oncology criteria. ${ }^{16}$

DNA for the assessment of $\mathrm{O}^{6}$-methylguanine-DNA methyltransferase (MGMT; by OncoMethylome's MGMT assay) and histone status were extracted from sections of formalin-fixed paraffin-embedded tissue blocks that were collected at the time of central pathology review.

\section{Safety}

Adverse events (AEs) were graded according to the National Cancer Institute Common Toxicity Criteria for Adverse Events (version 4.0). AEs of special interest were based on grouping AE terms by specific Medical Dictionary for Regulatory Activities baskets and standard Medical Dictionary for Regulatory Activities queries and included hypertension grade $\geq 3$; proteinuria grade $\geq 3$; GI perforation, abscesses, or fistula (any grade); wound-healing complications grade $\geq 3$; hemorrhage grade $\geq 3$ (any grade CNS bleeding; grade 2 hemoptysis); arterial thromboembolic events (any grade); venous thromboembolic events grade $\geq 3$; posterior reversible encephalopathy syndrome or reversible posterior leukoencephalopathy syndrome (any grade); congestive heart failure grade $\geq 3$; and non-GI fistula or abscess grade $\geq 2$.

\section{Statistical Analysis}

The sample size of this study was based on pragmatic considerations. A total enrollment of 120 patients was considered achievable over 3 years of accrual. Assuming an exponential model with a hazard ratio of 0.65 between the treatment groups-1-year event-free survival (EFS) of $30 \%$ in the RT+TMZ group and $46 \%$ in the bevacizumab (BEV) plus RT+TMZ group - the power of the log-rank test-two-sided, with an $\alpha$ of $5 \%$ - for a sample size of 60 patients per group was $60 \%$.

A prespecified interim futility analysis was performed on the basis of the first 60 randomly assigned patients who were observed for 1 year. If a protocol-specified threshold of a 10\% greater improvement in the 1-year EFS rate in the BEV plus RT+TMZ group versus the RT+TMZ group was not met, the study would be considered futile.

Prespecified sensitivity analyses included an unstratified analysis of CRRC-assessed EFS, and an unstratified analysis of CRRCassessed EFS with censoring of the data for patients who discontinued the study for any reason before experiencing an EFS event. We conducted exploratory multivariable Cox proportional hazards regression analyses to assess the impact of prognostic factors on EFS (age, bone age at baseline, high-grade glioma grade, complete resection or biopsy, MGMT gene promotor status, histone mutation status, Karnofsky performance status, and Lansky play-performance status). As an additional exploratory, post hoc sensitivity analysis, we assessed the heterogeneity of the effect of BEV according to stratification variables and other potential baseline prognostic factors in multivariable models, including interaction terms, shown in a forest plot. 


\section{RESULTS}

\section{Patients}

One hundred seventy-four patients were screened for enrollment, and 53 patients were excluded with screening. The most common reasons for screening failure included the failure to meet criteria, which included confirmation of local histologic diagnosis by a designated central reference neuropathologist $(n=17)$ and newly diagnosed localized, supratentorial or infratentorial cerebellar or peduncular, WHO grade III or IV gliomas $(n=9)$ after central radiologic review. Of patients, $5 \%$ had noneligible lowgrade glioma and were excluded.

\section{Patient Disposition and Protocol Deviations}

Patients received study treatment at 51 sites in 14 countries-Australia, Austria, Belgium, Canada, Czech Republic, Denmark, France, Hungary, Italy, the Netherlands, Poland, Spain, Sweden, and the United Kingdom.

There were 14 major protocol deviations (RT+TMZ [ $=8]$; BEV plus RT+TMZ [n=6]), including continued study treatment after experiencing progressive disease or an AE that warranted treatment discontinuation (RT+TMZ $[\mathrm{n}=8]$; BEV plus RT+TMZ $[n=5])$. One patient in the BEV plus RT+TMZ group underwent surgery before progressive disease. There was no crossover between treatment groups.

\section{Sensitivity Analyses of the Primary Efficacy End Point}

Results of sensitivity analyses performed on CRRC-assessed EFS were consistent with the primary analysis (Appendix Table A1, online only).

\section{Health-Related Quality of Life}

Mean baseline absolute scores for the Health Utility Index (HUI) questionnaire were balanced between the treatment groups ( 0.97 with RT+TMZ [ $\mathrm{n}=40]$ and 0.96 with BEV plus RT+TMZ [ $=46]$ ). At cycle 6 (day 1), mean changes from baseline in HUI score were -0.004 and 0.034 for the RT+TMZ $(n=24)$ and BEV plus RT+TMZ $(n=35)$ groups, respectively (a change in mean overall HUI score of 0.03 is considered a clinically meaningful difference; Horsman et al: Health Qual Life Outcomes. 1: 54, 2003); however, only four patients in the RT+TMZ group and three patients in the BEV plus RT+TMZ group completed the questionnaire at follow-up.

\section{Neuropsychological Function}

Neuropsychological function assessments were available for more than one half of patients during the treatment period, but availability decreased significantly for the follow-up period as a result of death and the withdrawal of consent. At end-of-treatment follow-up visit 1, assessments from 47 patients were available. Overall, patients for whom data were collected had normal cognitive function, an absence of motor deficit dysfunction, no seizures, an absence of raised intracranial pressure, and normal speech and language.

\section{Prognostic Factors}

Age, high-grade glioma grade (according to the WHO 2007 guidelines), and MGMT promoter status were not associated with an effect on EFS. Midline tumor location, biopsy only, and histone H3 K27M mutation were associated with lower EFS (Appendix Fig A1, online only). Multivariable Cox proportional hazards regression analysis revealed no significant association between potential baseline prognostic factors and EFS, but midline tumor location ( $v$ other) and a histone mutation at position G34 ( $v$ no mutation) were associated with poorer overall survival (Appendix Table A2, online only). 


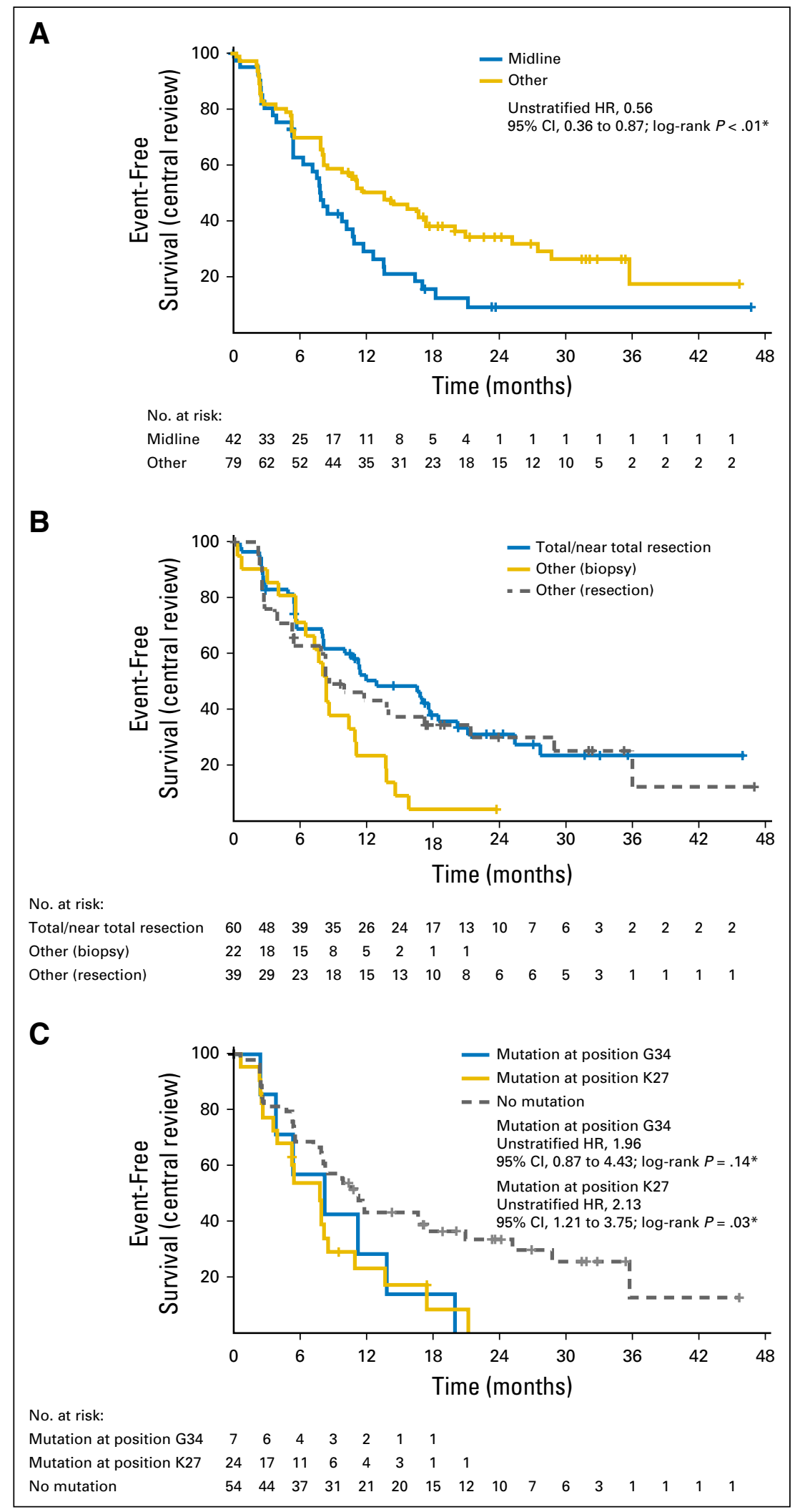

Fig A1. (A) Impact of tumor location, (B) extent of resection, and (C) histone mutation status on CRRC-assessed event-free survival. $\mathrm{Cl}$, confidence interval; CRRC, Central Radiology Review Committee; HR, hazard ratio. *A significance threshold of 0.01 was used for these analyses. 


\begin{tabular}{|c|c|c|}
\hline Sensitivity Analysis & $\begin{array}{l}R T+T M Z \\
(n=59)\end{array}$ & $\begin{array}{c}\text { BEV Plus RT+TMZ } \\
(n=62)\end{array}$ \\
\hline \multicolumn{3}{|l|}{ Unstratified analysis } \\
\hline Median, months & 11.8 & 8.2 \\
\hline $\mathrm{HR}(95 \% \mathrm{Cl})$ & \multicolumn{2}{|c|}{$1.18(0.77$ to 1.81$)$} \\
\hline \multicolumn{3}{|c|}{ Censoring for new anticancer therapy } \\
\hline Median, months & 11.8 & 8.2 \\
\hline $\mathrm{HR}(95 \% \mathrm{Cl})$ & \multicolumn{2}{|c|}{$1.44(0.90$ to 2.30$)$} \\
\hline \multicolumn{3}{|c|}{ Censoring for treatment discontinuation } \\
\hline Median, months & 16.4 & 14.5 \\
\hline $\mathrm{HR}(95 \% \mathrm{Cl})$ & \multicolumn{2}{|c|}{$1.41(0.80$ to 2.49$)$} \\
\hline
\end{tabular}

Abbreviations: BEV, bevacizumab; HR, hazard ratio; $R T$, radiotherapy; $T M Z$, temozolomide.

\begin{tabular}{|c|c|c|c|c|}
\hline Variable & Effect Size & $\mathrm{HR}$ & SE & $P$ \\
\hline \multicolumn{5}{|l|}{ CRRC-assessed EFS } \\
\hline \multicolumn{5}{|l|}{ Treatment } \\
\hline BEV plus $R T+T M Z$ & 0.047 & 1.048 & 0.287 & .8690 \\
\hline \multicolumn{5}{|l|}{ Age group, years } \\
\hline 3 to $\leq 5$ & -0.091 & 0.913 & 0.517 & .8603 \\
\hline 6 to $\leq 12$ & 0.331 & 1.393 & 0.299 & .2675 \\
\hline \multicolumn{5}{|l|}{ WHO-defined grade of HGG } \\
\hline IV & 0.332 & 1.393 & 0.315 & .2928 \\
\hline \multicolumn{5}{|l|}{ Resection } \\
\hline Other & 0.075 & 1.078 & 0.298 & .8010 \\
\hline Female sex & -0.215 & 0.806 & 0.264 & .4151 \\
\hline \multicolumn{5}{|l|}{ Tumor location } \\
\hline Midline & 0.595 & 1.812 & 0.485 & .2205 \\
\hline \multicolumn{5}{|l|}{ Histone status } \\
\hline Mutation at position G34 & 0.724 & 2.062 & 0.444 & .1027 \\
\hline Mutation at position K27 & 0.318 & 1.375 & 0.510 & .5325 \\
\hline \multicolumn{5}{|l|}{ OS } \\
\hline \multicolumn{5}{|l|}{ Treatment } \\
\hline BEV plus $R T+T M Z$ & -0.133 & 0.876 & 0.332 & .6900 \\
\hline \multicolumn{5}{|l|}{ Age group, years } \\
\hline 3 to $<6$ & -0.269 & 0.764 & 0.606 & .6571 \\
\hline 6 to $<13$ & 0.618 & 1.856 & 0.365 & .0899 \\
\hline \multicolumn{5}{|l|}{ WHO-defined grade of HGG } \\
\hline IV & 0.204 & 1.226 & 0.360 & .5719 \\
\hline \multicolumn{5}{|l|}{ Resection } \\
\hline Other & 0.228 & 1.256 & 0.339 & .5002 \\
\hline Female sex & -0.170 & 0.844 & 0.306 & .5792 \\
\hline \multicolumn{5}{|l|}{ Tumor location } \\
\hline Midline & 1.274 & 3.576 & 0.545 & .0193 \\
\hline \multicolumn{5}{|l|}{ Histone status } \\
\hline Mutation at position G34 & 1.167 & 3.212 & 0.498 & .0191 \\
\hline Mutation at position $\mathrm{K} 27$ & 0.067 & 1.069 & 0.547 & .9033 \\
\hline \multicolumn{5}{|c|}{$\begin{array}{l}\text { NOTE. Covariates included treatment ( } v \mathrm{RT}+\mathrm{TMZ} \text { ), age group ( } v 13 \text { to }<18 \\
\text { years), WHO-defined grade of HGG }(v \mathrm{lI} \text { ), resection complete or biopsy ( } v \text { total } \\
\text { or near total resection), sex ( } v \text { male), tumor location ( } v \text { other), and histone status } \\
\text { ( } v \text { no mutation). } \\
\text { Abbreviations: BEV, bevacizumab; CRRC, central radiology review committee; } \\
\text { EFS, event-free survival; HGG, high-grade glioma; HR, hazard ratio; OS, overall } \\
\text { survival; RT, radiotherapy; SE, standard error; TMZ, temozolomide. }\end{array}$} \\
\hline
\end{tabular}

